\title{
Seroprevalencia de brucelosis y leptospirosis en pobladores urbanos con crianza traspatio en el distrito de José Leonardo Ortiz de Chiclayo
}

Seroprevalence of brucellosis and leptospirosis in urban citizens with backyard breeding in the district of José Leonardo Ortiz, Chiclayo

Luis A. Yamunaqué-Castro 1,a, Franklin R. Aguilar-Gamboa 2,a, Eyner A. Quenema-Díaz 2,b, Lizzie K. Becerra-Gutiérrez ${ }^{2,3, c}$, Heber Silva-Díaz ${ }^{3,4, c}$

\section{RESUMEN}

Objetivo: Describir la seroprevalencia de brucelosis y leptospirosis y los factores asociados, en pobladores urbanos con crianza traspatio en el distrito de José Leonardo Ortiz de Chiclayo, Perú. Material y métodos: Estudio prospectivo, transversal y analítico realizado en 90 personas durante los meses de octubre a diciembre del 2016. Se determinaron anticuerpos IgM e IgG específicos para brucelosis y leptospirosis mediante la técnica de ELISA indirecto. Los factores asociados se recolectaron usando una encuesta estructurada. Resultado: La seroprevalencia de brucelosis fue $2,2 \%$ y de leptospirosis $28,9 \%$. Las características ambientales más frecuentes en la población fueron, disposición de excretas en letrinas $(73,3 \%)$, contacto con agua estancada $(83,3 \%)$ contacto con roedores $(62,2 \%)$. No se encontró asociación entre los factores evaluados y las enfermedades. Conclusiones: Se evidencia una alta seroprevalencia de leptospirosis y baja para brucelosis en personas con crianza traspatio del distrito de José Leonardo Ortiz de Chiclayo, revelando un problema de salud pública vigente. Se recomienda continuar con estudios longitudinales que permitirían evaluar factores de riesgo y realizar intervenciones preventivas.

PALABRAS CLAVE: Leptospirosis, brucelosis, crianza de animales domésticos, estudios seroepidemiológicos, salud urbana. (Fuente: DeCS-BIREME).

\section{SUMMARY}

Objective: To describe the seroprevalence of and risk factors for brucellosis and leptospirosis in urban citizens with backyard breeding in the district of José Leonardo Ortiz, Chiclayo. Methods: Cross-sectional study including 90 citizens during the month of October and December of 2016. IgM and IgG specific antibodies for brucellosis and leptospirosis were determined using the indirect ELISA method. Factors associated with these diseases were gathered using a structural survey. Results: The seroprevalence of brucellosis and leptospirosis was $2.2 \%$ and $28.9 \%$, respectively. The most common environmental features of the population were that $73.3 \%$ use latrines

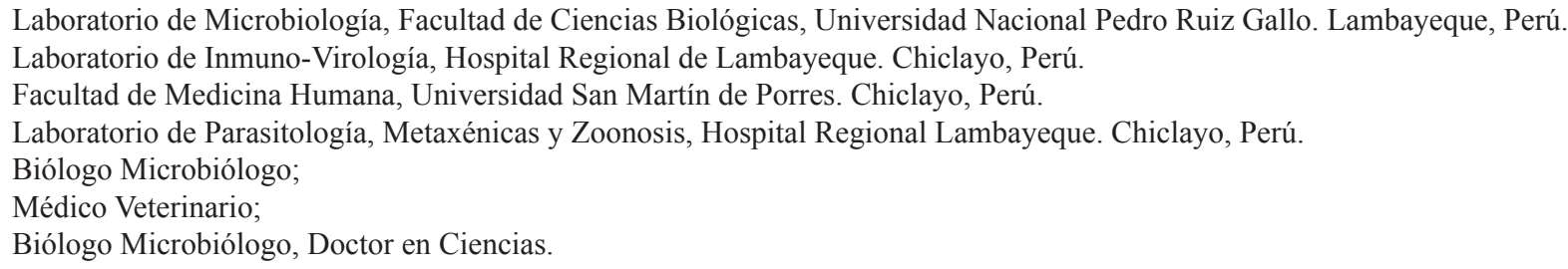


83.3\% had contact with stagnant water and $62.2 \%$ had contact with rodents. No association between the variables looked for and these two diseases was found. Conclusions: We found a high seroprevalence of leptospirosis but a low prevalence of brucellosis in this setting. We suggest performing longitudinal studies that may identify risk factors to prevent these diseases.

KEYWORDS: Leptospirosis, brucellosis, seroprevalence, animal husbandries, urban health. (Source: MeSHNLM).

\section{INTRODUCCIÓN}

La brucelosis y la leptospirosis son enfermedades zoonóticas bacterianas emergentes y de distribución mundial, reportándose al año 0,5 y 1,03 millones de casos nuevos respectivamente a nivel global (1-4), generando gran impacto económico debido a los costos en el tratamiento, hospitalización, ausentismo laboral, perdidas en la producción ganadera y baja productividad en el ganado, además de la alta tasa de mortalidad que ocasiona la leptospirosis $(4,5)$.

La brucelosis es causada por especies del género Brucella (B. melitensis B. abortus y B. suis), cuyos principales reservorios son el ganado caprino, bovino y porcino, respectivamente. En América del Sur la brucelosis humana está excluida en casi todos los países con excepción de Argentina y Perú. En este último con una tasa de incidencia de 3,49/100 000 habitantes (2); su incremento se ha relacionado con el movimiento de animales caprinos de la sierra a la costa por actividades de pastoreo (6), así como con el aumento de la incidencia bovina y caprina (7).

La leptospirosis se adquiere por contacto con animales o sus productos contaminados con especies patógenas del género Leptospira. En el continente americano se notificaron 10433 casos de leptospirosis humana en el 2014, con una tasa de incidencia de 2,11/100 000 habitantes. El Perú presentó una frecuencia de $22 \%$ de casos y una tasa de incidencia más alta en América del Sur de 7,6/100 000 habitantes (8).

La seroprevalencia de brucelosis descrita para la región de Lambayeque es de 10,7\% en trabajadores de mataderos (9). En cuanto a la leptospirosis se ha reportado prevalencias de $64,6 \%$ para pobladores con exposición ocupacional en la región San Martin (10); $19,91 \%$ a $26,6 \%$ en pacientes febriles en Lambayeque $(11,12)$.
Estas dos infecciones comparten contexto epidemiológico, registrándose casos de coinfección en áreas donde existen factores epidemiológicos comunes, complicando el pronóstico, diagnóstico y tratamiento (1). Afectan a grupos ocupacionales como veterinarios, matarifes $(13,14)$, ganaderos $(15,16)$, agricultores $(10,14)$ y amas de casa $(11,17)$; quienes mantienen contacto con animales infectados (ganado bovino, porcinos, caprinos, ovinos, perros y roedores), fetos abortados, secreciones genitales u orina $(4,18)$, leche y sus derivados no pasteurizados $(4,17)$ y aguas contaminadas $(19,20)$. Esta última asociada principalmente con leptospirosis en actividades recreacionales (13), agricultores de arroz (10) e inundaciones $(13,16)$.

En el distrito de José Leonardo Ortiz de Chiclayo, existen zonas urbanas recientemente pobladas principalmente por inmigrantes de la región andina de Cajamarca. La mayoría de estas familias han improvisado crianzas traspatio en el afán de recrear sus actividades económicas previas que, junto a existencia parcial de servicios básicos, han generado las condiciones para el desarrollo de enfermedades.

El objetivo del estudio fue determinar la seroprevalencia y factores asociados de la leptospirosis y brucelosis en pobladores urbanos con crianza traspatio en el distrito de José Leonardo Ortiz de Chiclayo.

\section{MATERIAL Y MÉTODOS}

Estudio observacional, analítico, prospectivo, realizado durante los meses de octubre a diciembre del 2016. La población de estudio estuvo constituida por pobladores de los sectores de Culpón, Santa Ana y Miraflores del distrito de José Leonardo Ortiz, provincia de Chiclayo, Lambayeque.

La muestra estuvo conformada por 90 pobladores, calculada para estimar una proporción en una 
Tabla 1. Características sociodemográficas, ambientales y de saneamiento de los pobladores urbanos con crianza traspatio en el distrito de José Leonardo Ortiz de Chiclayo, octubre a diciembre del 2016 (n: 90).

\begin{tabular}{|c|c|c|c|}
\hline Variable & n $(\%)$ & Lepto-IgM positivo/Total (\%) & $p$ \\
\hline \multicolumn{4}{|l|}{ Género } \\
\hline Femenino & $45(50,0)$ & $16 / 45(35,6)$ & $0,1736^{*}$ \\
\hline Masculino & $45(50,0)$ & $10 / 45(22,2)$ & \\
\hline \multicolumn{4}{|l|}{ Grupo etario (años) } \\
\hline$<18$ & $16(17,8)$ & $3 / 16(18,8)$ & $0,5871 ”$ \\
\hline $18-59$ & $63(70,0)$ & $20 / 63(31,8)$ & \\
\hline$>59$ & $11(12,2)$ & $3 / 11(27 / 3)$ & \\
\hline \multicolumn{4}{|l|}{ Nivel de instrucción } \\
\hline Primaria & $49(54,4)$ & $15 / 49(30,6)$ & $0,7024 ”$ \\
\hline Secundaria & $33(36,7)$ & $8 / 33(24,2)$ & \\
\hline Superior & $8(8,9)$ & $3 / 8(37,5)$ & \\
\hline \multicolumn{4}{|l|}{ Ocupación } \\
\hline Ama de casa & $33(36,7)$ & $13 / 33(39,4)$ & $0,2441 ”$ \\
\hline Comerciante-Estudiante & $23(25,6)$ & $5 / 23(21,7)$ & \\
\hline Ganadero-Obrero & $34(37,8)$ & $8 / 34(23,5)$ & \\
\hline \multicolumn{4}{|l|}{ Disposición de excretas } \\
\hline Red & $24(26,7)$ & $9 / 24(37,5)$ & $0,3014^{*}$ \\
\hline Letrina & $66(73,3)$ & $17 / 66(25,8)$ & \\
\hline \multicolumn{4}{|l|}{ Fuente de consumo de Agua } \\
\hline Pozo comunitario & $13(14,4)$ & $6 / 13(46,2)$ & $0,1856^{*}$ \\
\hline Red pública & $77(85,6)$ & $20 / 77(26,0)$ & \\
\hline \multicolumn{4}{|l|}{ Contacto con agua estancada } \\
\hline $\mathrm{Si}$ & $75(83,3)$ & $22 / 75(29,3)$ & $>0,9999 *$ \\
\hline No & $15(16,7)$ & $4 / 15(26,67)$ & \\
\hline \multicolumn{4}{|l|}{ Presencia de roedores } \\
\hline $\mathrm{Si}$ & $56(62,2)$ & $15 / 56(26,8)$ & $0,6349^{*}$ \\
\hline No & $34(37,8)$ & $11 / 34(32,35)$ & \\
\hline \multicolumn{4}{|c|}{$\begin{array}{l}\text { Contacto con vísceras y fluidos } \\
\text { animales }\end{array}$} \\
\hline $\mathrm{Si}$ & $46(51,1)$ & $14 / 46(30,4)$ & $0,8180^{*}$ \\
\hline No & $44(48,9)$ & $12 / 44(27,3)$ & \\
\hline \multicolumn{4}{|c|}{ Consumo de lácteos no pasteurizados } \\
\hline $\mathrm{Si}$ & $23(25,6)$ & $8 / 23(34,8)$ & \\
\hline No & $67(74,4)$ & $18 / 67(26,9)$ & $0,5945^{*}$ \\
\hline \multicolumn{4}{|l|}{ Actividades recreativas } \\
\hline Sin antecedentes & $57(63,3)$ & $13 / 57(22,8)$ & $0,1467^{*}$ \\
\hline Rio-Dren & $33(36,7)$ & $13 / 33(39,4)$ & \\
\hline
\end{tabular}

$p^{*}$, Irwin-Fisher bilateral; $p$ ", Chi Cuadrado Pearson.

población desconocida, a un nivel de confianza al $95 \%$, proporción esperada $64,6 \%(10)$, y error $10 \%$ (Win-Epi, Working in Epidemiology). Los criterios de inclusión fueron: residencia permanente en los sectores de estudio, no haber viajado fuera de la región en los últimos tres meses y aceptación de participar en el estudio. Los criterios de exclusión fueron: personas que presentaron alguna enfermedad o condición que dificulte la toma de muestra.

El muestreo fue no probabilístico de tipo consecutivo, considerando a todos los integrantes de 


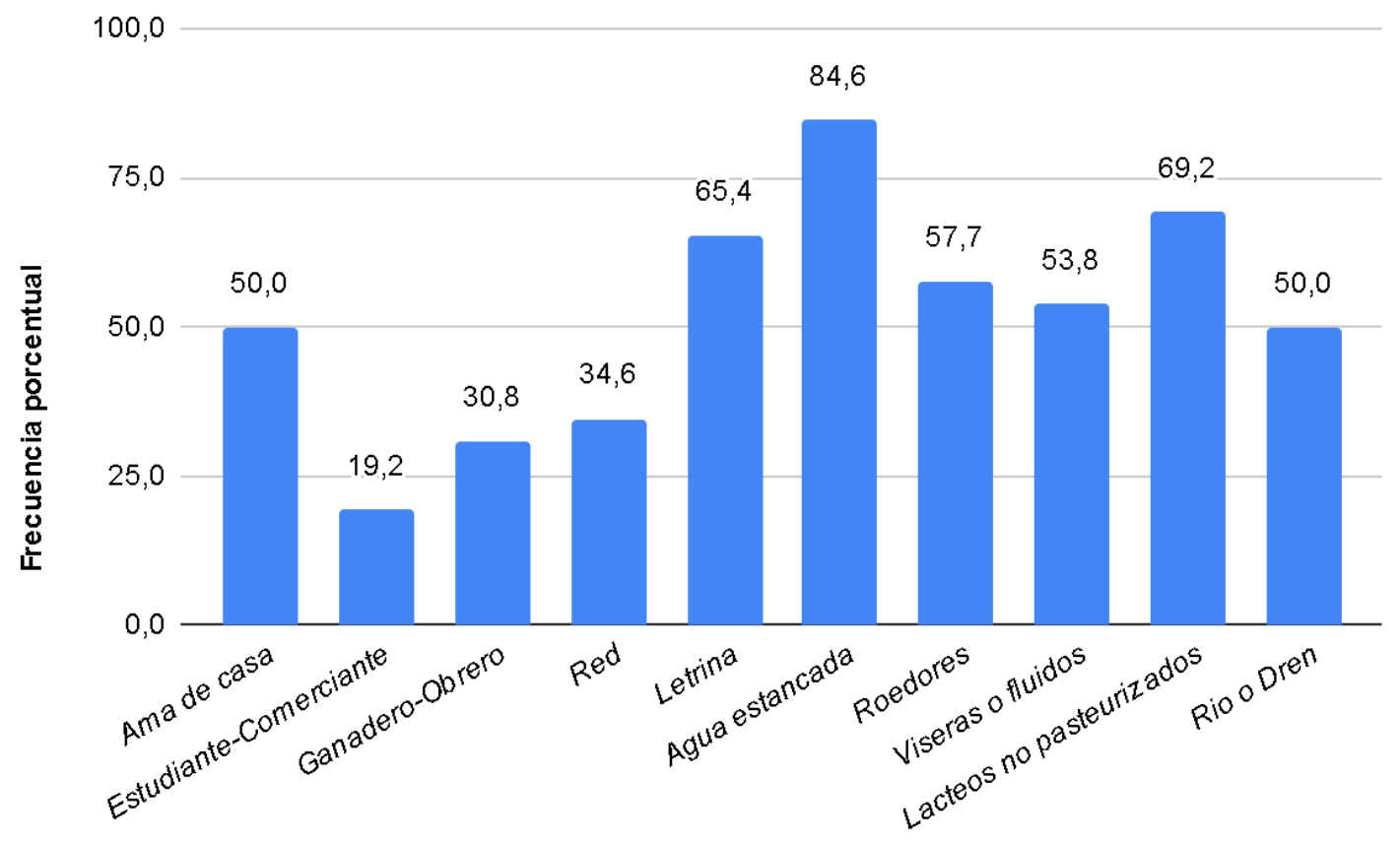

Gráfico 1. Distribución de las características sociodemográficas y ambientales de los casos de leptospirosis en pobladores urbanos con crianza traspatio en el distrito de José Leonardo Ortiz de Chiclayo, octubre a diciembre del 2016

las viviendas con crianza traspatio que cumplían con los criterios de selección.

La determinación de la seroprevalencia de la brucelosis y leptospirosis se realizó mediante la detección cuantitativa de anticuerpos IgM e IgG antiBrucella, e IgM anti-Leptospira, respectivamente; por la técnica ELISA indirecta. Se utilizaron kits comerciales. Se siguieron las recomendaciones del fabricante en el protocolo de ensayo de Elisa. Los factores asociados se recolectaron usando una encuesta estructurada.

Se utilizó estadística descriptiva para las variables cualitativas y cuantitativas, considerando frecuencias absolutas y relativas para las primeras y medias o medianas para las segundas, según su distribución. El análisis bivariado para establecer la asociación entre los factores y la prevalencia de las enfermedades se realizó mediante la prueba de Chi cuadrado o prueba exacta de Fisher. Se utilizaron los programas informáticos SPSS v. 22.0 e Infostat 2008.

El estudio fue revisado y aprobado por el Comité de Ética en Investigación del Hospital Regional Lambayeque (HRL), con número de registro 0214031-16. La participación en el estudio fue voluntaria y aceptada a través de la firma de un consentimiento informado. Los participantes que resultaron positivos a leptospirosis y brucelosis fueron derivados al servicio del programa de Metaxénicas y Zoonosis del HRL, con el fin de ser atendidos por un médico infectólogo y recibir un tratamiento apropiado.

\section{RESULTADOS}

Participaron 90 personas de los sectores Culpón, Santa Ana y Miraflores del distrito de José Leonardo Ortiz, Chiclayo, Lambayeque. La muestra se caracterizó por poseer proporción equitativa en cuanto al sexo, y una media de edad de 37,7 \pm 7,9 años. Las características sociodemográficas y ambientales se describen en la tabla 1.

La seroprevalencia de brucelosis fue $2,2 \%$ (IC 95\%: $0,0-5,3$ ) y de leptospirosis $28,9 \%$ (IC $95 \%: 19,5$ $-38,3)$. Los dos casos positivos por la técnica de Elisa de brucelosis fueron confirmados mediante la prueba de Rosa de Bengala y aglutinaciones en tubo. Estos casos pertenecieron a adultos: ama de casa y agricultor; quiénes manifestaron contacto con vísceras, agua estancada y roedores; además uno de ellos presentó coinfección para brucelosis y leptospirosis.

El análisis bivariado no mostró asociación de factores sociodemográficos y ambientales con la 
leptospirosis (tabla 1). La distribución porcentual de las características sociodemográficas y ambientales de los casos de leptospirosis se presentan en el gráfico 1 . La mínima cantidad de casos de brucelosis encontrada en el estudio no permitieron evaluar los factores asociados.

\section{DISCUSIÓN}

La crianza traspatio de animales domésticos en los sectores más vulnerables del distrito de José Leonardo Ortiz de la ciudad de Chiclayo representa un riesgo para la aparición de brotes por enfermedades zoonóticas como la brucelosis y leptospirosis, generando la necesidad de describir la seroprevalencia y los factores asociados que se presentan en este contexto.

La seroprevalencia de brucelosis hallada concuerda con las tasas de incidencia reportadas para los países sudamericanos (2) y similares a estudios realizados en trabajadores de fincas y mataderos en Ecuador (15). Estos valores relativamente bajos de brucelosis en Perú se han atribuido a zonas geográficas con limitado acceso, en donde el movimiento de animales es nulo, formando una barrera para la entrada de está zoonosis (3).

En Lambayeque se ha encontrado una seroprevalencia de brucelosis de $10,4 \%$ en trabajadores de mataderos (8). La diferencia observada con este estudio se debe posiblemente al elevado riesgo de infección por Brucella spp., que existe debido a la permanente exposición a sangre de animales de distintos lugares que presentan los trabajadores de mataderos. Sin embargo, existen otros factores de riesgo para una alta prevalencia como la presencia de ganado caprino, derivados lácteos no pasteurizados de origen caprino y pastoreo migratorio de costa a sierra $(6,21)$, factores que no se presentaron en la población estudiada.

La presencia de dos casos de brucelosis, aunque representa baja prevalencia, es un hallazgo importante para la epidemiología de esta enfermedad en región por cuanto no existen reportes de esta enfermedad en este tipo de población. Sin embargo, teniendo en cuenta la exposición ambiental y ocupacional que presenta la población de estudio y que son favorables a la enfermedad, es posible estos hallazgos. No obstante, es importante ampliar el estudio en una mayor cantidad de personas.
Los datos reportados para la leptospirosis en el Perú son variables y dependen de la región, grupo de riesgo o factores ambientales. En la región de Lambayeque la seroprevalencia de esta zoonosis se encuentra entre $19,9 \%$ y $26,6 \%$ en pacientes febriles, utilizando pruebas de microaglutinación en tubo (MAT) y ELISA IgM respectivamente $(11,12)$. Estos resultados concuerdan con la seroprevalencia obtenida en el estudio.

Se han reportado seroprevalencias más elevadas en grupos con riesgo ocupacional, es así que en San Martin la seroprevalencia de leptospirosis en agricultores de arroz fue de 64,6\% utilizando como prueba diagnóstica MAT, y de $15,0 \%$ por ELISA IgM (10); la diferencia de seroprevalencia con este estudio se puede explicar por la sensibilidad de las pruebas utilizadas; no obstante, el tiempo de exposición a agua estancada, las lesiones que sufren constantemente en la planta de los pies por trabajar descalzo, la presencia y manipulación de roedores, son factores determinantes para que los resultados obtenidos en agricultores de arroz sean diferentes y mayores a los hallados en los pobladores con crianza traspatio (10).

A pesar que el análisis bivariado no mostró asociación entre algún factor estudiado y la leptospirosis, debido probablemente al reducido tamaño de muestra, se resalta la alta frecuencia de factores de riesgo en la población; como la falta de desagües, contacto con agua estancada, presencia de roedores, contacto con vísceras y recrearse en ríos o dren, que son características reconocidas y aceptadas por su relación con la enfermedad.

De los casos positivos la mayor seroprevalencia se encontró en amas de casa (50\%), seguido de Ganaderoobrero (30,8\%), estudiante comerciante $(19,2 \%)$, esta mayor frecuencia en amas de casa y ganaderos ha sido registrada para la región Lambayeque (11). Las amas de casa y ganaderos-obreros en la población en estudio realizaban tareas de limpieza, alimentación y cuidado de los animales, exponiéndose a mayor contacto con heces u orina aumentando el riesgo de leptospirosis. Por otro lado, el $84 \%$ de estos casos estaban en contacto con agua estancada, factor que ha sido asociado a leptospirosis y que sería una fuente probable para que se presente brotes hiperendémicos ante inundaciones $(16,22)$, como lo sucedido durante el "El Niño Costero" periodo en el cual se presentó un incremento exponencial en las regiones del norte del Perú (23). 
El estudio tuvo algunas limitaciones: en el caso de la leptospirosis, la seroprevalencia pudo ser sobre o subestimada debido a sesgo de medición; por cuanto, por razones de acceso no pudo tomarse segunda muestra para evaluar seroconversión, y por limitado presupuesto, no se realizó la confirmación por la técnica de microaglutinación (MAT). Del mismo modo, la seroprevalencia de la brucelosis pudo haber tenido sesgo de medición debido al uso general de un solo método diagnóstico, restringiendo solo a los positivos la confirmación con Rosa de Bengala y aglutinación en tubo. En ambas enfermedades el diseño de investigación tipo transversal y el tamaño de muestra no permitieron establecer causalidad.

Se concluye que pobladores con crianza traspatio y que residen en zona urbana del distrito de José Leonardo Ortiz, Chiclayo, presentan alta seroprevalencia de leptospirosis; y baja seroprevalencia de brucelosis, en una zona considerada no endémica para esta enfermedad. No se pudo establecer los factores asociados, pero la presencia de roedores, contacto con agua estancada y falta de alcantarillado caracterizaron el pobre saneamiento ambiental de la población.

Se recomienda continuar con estudios longitudinales que permitirían evaluar factores de riesgo y realizar intervenciones preventivas, además se sugiere tomar acciones de políticas públicas para mejorar el saneamiento ambiental en el lugar y reducir este problema de salud pública vigente en la región; y que sin embargo, podría agravarse ante la presencia de inundaciones u otros desastres naturales.

\section{Agradecimientos:}

Los autores desean dar un especial agradecimiento a Jhosbelly Guadalupe Vásquez y Frank Tene Vargas, por el apoyo en la etapa de muestreo.

\section{Declaración de Financiamiento y de conflictos de interés:}

Los reactivos de laboratorio y viáticos para el muestreo fueron autofinanciados. El uso del Laboratorio de Investigación fue subvencionado por la Dirección de Investigación del Hospital Regional Lambayeque. Los autores declaran no tener conflictos de interés.

\section{Contribución de autoría:}

LAYC: Participó en el análisis y discusión de los datos, revisión bibliográfica, redacción del manuscrito, preparación del manuscrito y aprobación de la versión final. FRAG: Participó en la concepción de la idea de trabajo, recolección de datos, revisión crítica y aprobación de la versión final EAQD: Participó en la recolección de datos, revisión crítica del artículo y aprobación de la versión final del manuscrito. LKBG: Participó en la recolección de datos, revisión crítica del artículo y aprobación de la versión final del manuscrito. HSD: Participó en el diseño de estudio, análisis de datos, redacción del manuscrito y aprobación de la versión final del manuscrito.

\section{Correspondencia:}

Luis Antonio Yamunaqué Castro

Pro. Augusto B. Leguía Nro. 100 (esquina con Av. Progreso $\mathrm{N}^{\mathrm{o}} 110$-120)

Chiclayo, Lambayeque, Perú.

Teléfono: 51996484081

Correo electrónico: luisyamunaquecastro@gmail.com

\section{REFERENCIAS BIBLIOGRÁFICAS}

1. Jaramillo L, Arboleda M, García V, Agudelo-FlórezP. Coinfección brucelosis-leptospirosis, Urabá, Colombia: Reporte de caso. Infectio. 2014; 18(2):726.

2. Pappas G, Papadimitriou P, Akritidis N, Christou L, Tsianos E V. The new global map of human brucellosis. Lancet Infect Dis. 2006; 6(2):91-9.

3. Meza CA, Calle ES, Manchego SA, Angulo JC, Morales CS, Ara GM. Seroprevalencia de brucelosis bovina en el distrito de Puerto Inca, Huánuco. Rev Investig Vet del Perú. 2015; 21(2):223-6.

4. Barreto G, Rodríguez C. La leptospirosis en las producciones caprinas. Rev prod anim. 2018; 30(3):57-62.

5. Guzmán-Hernández RL, Contreras-Rodríguez A, Ávila-Calderón ED, Morales-García MR. Brucelosis: zoonosis de importancia en México. Rev Chil infectología. 2016; 33(6):656-62.

6. Zapatel J, Málaga H. Epidemiologia de la brucelosis caprina en el Perú. Bol Oficina Sanit Panam. 1971; 71(2): 121-131. (Citado el 15 de mayo del 2019). Disponible en: http://iris.paho.org/xmlui/ handle/123456789/10978

7. Méndez-Lozano M, Rodríguez-Reyes EJ, SánchezZamorano LM. Brucelosis, una zoonosis presente en la población: Estudio de series de tiempo en México. Salud Publica Mex. 2015; 57(6):519-27.

8. Pan American Health Organization. Leptospirosis: Region of the Americas. Washington: Pan American Health Organization; 2015. (Citado el 15 de mayo del 2019). Disponible en: https://www.paho.org/hq/ dmdocuments/2016/2015-PHE-Leptospirosis- 
Profile-Americas.pdf

9. Santa-Cruz CY, Vásquez-del-Castillo AM. Seroprevalencia de brucelosis en trabajadores de mataderos en la región de Lambayeque, Perú. Rev Exp en Med. 2019; 5(1):93-9.

10. Alarcon-Villaverde JO, Romani-RomaniF, Tejada RA, Wong-Chero P, Céspedes-Zambrano M. Seroprevalencia de leptospirosis y caracteristicas asociadas en agricultores de arroz de una región tropical del Perú. Rev Peru Med Exp Salud Publica. 2014; 31(2):195-203.

11. Silva-Díaz H, Llatas-Cancino DN, Campos-Sánchez MJ, Aguilar-Gamboa FR, Mera-Villasis KM, Valderrama-Ayén MY. Frecuencia de leptospirosis y características socio-demográficas en pacientes febriles del norte del Perú. Rev Chil infectología. 2015; 32(5):530-5.

12. Pimentel JG, Vásquez L. Prevalencia de Leptospira interrogans y serovariedades en pacientes con síndrome febril en el distrito de Olmos-Lambayeque. Marzo-septiembre 2016. Tesis de Licenciatura. Lambayeque, Perú: Universidad Nacional Pedro Ruiz Gallo; 2017. 84 p.

13. Pulido-Villamarín A, Carreño-Beltrán G, MercadoReyes M, Ramírez-Bulla P. Situación epidemiológica de la leptospirosis humana en Centroamérica, Suramérica y el Caribe. Univ Sci. 2014; 19(3):24764.

14. Lemos TS, Cequinel JC, Costa TP, et al. Outbreak of human brucellosis in Southern Brazil and historical review of data from 2009 to 2018. PLoS Negl Trop Dis. 2018; 12(9):1-12.

15. Zambrano MD, Pérez M. Seroprevalencia de brucelosis en ganado bovino y en humanos vinculados a la ganadería bovina en las zonas norte y centro de la provincia Manabí, Ecuador. Rev Salud Anim. 2015; 37:164-172.
16. Donaires LF, Céspedes MJ, Sihuincha MG, Pachas PE. Determinantes ambientales y sociales para la reemergencia de la leptospirosis en la región amazónica del Perú, 2012. Rev Peru Med Exp Salud Publica. 2012; 29(2):280-4.

17. Cavalcanti C, Almeida JA, Feitosa A, Firmino S, Andrade MV, Silva-Júnior F. Prevalence of Brucella spp in humans. Rev Lat Am Enfermagem. 2015; 23(5):919-26.

18. Valle T, Lago Y, García I. Resultados de intervención educativa sobre brucelosis humana en zonas de riesgo de enfermar en Argelia. Rev cienc méd Pinar Río. 2018; 22(4):804-14.

19. Benavides B, Jiménez E. Factores de riesgo asociados a la seroprevalencia de brucelosis y leptospirosis en los operarios de la planta de beneficio de Pasto, Nariño. Revista Univ y Salud. 2012; 1:42-9.

20. Sánchez L, García CJ, Mattar S, Rodríguez I. Seroprevalencia de leptospirosis en pacientes con síndrome febril no palúdico. Rev Cubana Med Trop. 2018; 70(1):45-54.

21. García ML, Herrera ME, Pérez AM, Castillo L, Kestler RO. Seroprevalencia de leptospirosis humana en un asentamiento del área urbana de la ciudad de Guatemala. Rev Cubana Med Trop. 2013; 65(2):16676.

22. Ghizzo J, Nazário NO, Freitas PF, et al. Temporal analysis of the relationship between leptospirosis, rainfall levels and seasonality, Santa Catarina, Brazil, 2005-2015. Rev Inst Med Trop Sao Paulo. 2018; 60:1-9.

23. Lecca-Bartra A, Oblitas-Campos R, Leguía J. Leptospirosis después del "Niño Costero": una visión actual del norte peruano. Rev Chilena Infecto. 2017; 34(4):418-418.

Recibido: 01/07/2019

Aceptado: 09/01/2020 